\title{
Lipiodol retention pattern assessed by cone beam computed tomography during conventional transarterial chemoembolization of hepatocellular carcinoma: accuracy and correlation with response
}

Jungang Hu${ }^{1}$, Majid Maybody², Guang Cao ${ }^{1}$, Xiao Wang ${ }^{3}$, Hui Chen ${ }^{1}$, Xu Zhu' ${ }^{1}$, Renjie Yang ${ }^{1}$ and Xiaodong Wang ${ }^{{ }^{*}}$

\begin{abstract}
Background: To investigate accuracy of intraprocedural cone beam computed tomography (CBCT) compared to fluoroscopy for detection of lipiodol retention pattern during conventional transarterial chemoembolization (CTACE) of hepatocellular carcinoma (HCC) and its correlation with short-term response.

Methods: Between September 2013 and July 2014, 29 patients with HCC underwent chemoembolization of 51 tumors (mean diameter $28.1 \mathrm{~mm}$, range 10.0-136.3 mm). Lipiodol retention pattern was assessed by CBCT at the endpoint of CTACE compared by fluoroscopy. Depending on the pattern of tumor covered by lipiodol three classes were defined: complete (more than $90 \%$, no peripheral defects), moderate (50-90\%, some with or without peripheral defects), and poor (less than $50 \%$ ). Tumor response was assessed by modified Response Evaluation Criteria in Solid Tumors (mRECIST) based on follow-up contrast enhanced (CE) computed tomography (CT) or magnetic resonance imaging (MRI) obtained 4-6 weeks post-CTACE. Correlations between lipiodol retention patterns on CBCT and fluoroscopy as well as tumor response were assessed using multivariate logistic regression.

Results: Of 51 hepatic tumors, 40 (78.4\%) had complete response (CR); 8 (15.7 \%) had partial response (PR); 1 (2.0\%) had stable disease (SD); and 2 (3.9\%) had progressive disease (PD). The degree of lipiodol retention scored excellent, moderate, and poor, in fluoroscopic images vs CBCT images were 23 (45.1\%) vs 39 (76.5 \%), 19 (37.3 \%) vs 11 (21.6 \%), and $9(17.6 \%)$ vs $1(2.0 \%)$, respectively. Lipiodol retention assessment with $C B C T\left(A_{z}=0.75\right)$ is more accurate than fluoroscopy $\left(A_{z}=0.54\right)$ in predicting target tumor response. Other than lipiodol retention pattern assessed with CBCT $(p=0.01)$, tumor size $(p=0.04)$ is an independent predictors of $C R$.
\end{abstract}

Conclusion: CBCT is more accurate than fluoroscopy in classification of lipiodol retention pattern in HCC tumors at the time of CTACE. CBCT could be used as a reliable intra precedural monitoring modality of CTACE.

Keywords: Cone beam computed tomography, Fluoroscopy, Transarterial chemoembolization, Hepatocellular carcinoma, Liver

\footnotetext{
* Correspondence: xiaodongw75@yahoo.com

${ }^{1}$ Department of Interventional Radiology, Peking University Cancer Hospital \&

Institute, Key laboratory of Carcinogenesis and Translational Research

(Ministry of Education), Beijing 100142, China

Full list of author information is available at the end of the article
} 


\section{Background}

Hepatocellular carcinoma (HCC) is the sixth most common cancer and the third leading cause of cancerrelated-death worldwide [1]. Curative treatments including resection, liver transplantation and local ablation are indicated in less than $30 \%$ of patients at the time of diagnosis [2]. Transarterial chemoembolization (TACE) is the current standard of care for HCC patients with unresectable intermediate-stage disease and has been reported to prolong survival [3-5].

Early assessment of the effectiveness of TACE and identifying predictors of tumor response are crucial for successful management. Intraprocedural image monitoring is important to assess the endpoint of TACE and application of additional treatment if needed while the patient is still in the angiography suite.

cTACE uses iodized oil (lipiodol) (Guerbet, Aulnay-sousBois, France) as a carrier of chemotherapeutic agents [6, 7] and the degree of intratumoral lipiodol retention has been shown to correlate with tumor necrosis and local tumor recurrence with $\mathrm{HCC}[8,9]$.

Visualizing distribution of lipiodol after chemoembolization by fluoroscopy or computed tomography (CT) scan ensures tumor targeting [10]. However, fluoroscopic imaging may fail to detect lack of lipiodol accumulation within the tumor [11]. Computed tomography is more accurate to depict cross-sectional lipiodol distribution [12], but it is cumbersome to transfer patients from the angiography suite to the CT suite since the hybrid angiography-CT systems are not always available.

Cone beam computed tomography (CBCT) using a flat-panel detector is increasingly used and widely available [13]. It has been shown to have lipiodol detection rate comparable with multidetector CT imaging [14]. Lipiodol retention with the help of three dimensional quantification software was found to correlate with tumor response in $\mathrm{HCC}$ in a recently published paper [15]. But this software is not widely available.

The goal of this study was to investigate whether gross lipiodol retention pattern on CBCT imaging immediately after cTACE of hepatocellular carcinoma can be used as predictor of tumor response by mRECIST, and whether this could be used as a reliable intra procedural monitoring modality.

\section{Methods}

\section{Subjects}

Authorization from the Institutional Ethics Committee was not needed for this retrospective study in our center. Informed consent was obtained from each patient. All cTACE interventions performed between September 2013 and July 2014 were reviewed. From the total of 189 patients, the following were excluded: non HCC etiology $(n=38)$, prior cTACE $(n=98)$, other treatments within
4 weeks prior to CTACE $(n=3)$, lack of CBCT during cTACE $(n=12)$, significant image artifacts on CBCT $(n=3)$, poor quality follow-up CT or MRI imaging $(n=2)$ and those lost to follow-up $(n=4)$. The study group included all patients $(n=29)$ with HCC who were eligible to undergo their first cTACE as described below, whose cTACE included CBCT, who had not undergone systemic therapy within one month prior to CTACE until the first follow-up imaging study 4-6 weeks after cTACE, who had undergone dynamic contrast-enhanced MR or CT imaging within one month before cTACE and had follow up imaging 4-6 weeks after cTACE.

The diagnosis of HCC was confirmed by biopsy or by characteristic radiologic findings for tumors larger than one $\mathrm{cm}$ in patients at risk for HCC such as cirrhotics or hepatitis B carriers. Eligibility criteria for cTACE were as follows: Eastern Cooperative Oncology Group (ECOG) performance status $\leq 2$; Child-Pugh classification A or B; tumor involvement less than $60 \%$ of total liver volume; absence of portal vein tumor thrombus; absence of ascites; albumin > $2.5 \mathrm{~g} / \mathrm{dl}$; alanine aminotransferase and aspartate aminotransferase $<5$ times the upper normal limit; total serum bilirubin $<3.0 \mathrm{mg} / \mathrm{dl}$; serum creatinine $<2.0 \mathrm{mg} / \mathrm{dl}$; platelet count $>50,000 / \mathrm{mm}^{3}$ and international normalized ratio (INR) $\leq 1.5$. Patient demographics and tumor data are listed in Table 1.

\section{Transarterial chemoembolization}

All cTACE were performed using a $40 \mathrm{~cm}$ flat panel angiography system (Innova 4100, GE Healthcare, Waukesha, WI, USA). The technique for embolization has been previously described $[4,16]$. Details of the hepatic artery anatomy, feeding artery and location of tumors, and portal vein patency and flow direction were obtained from hepatic and superior mesenteric arteriography via a 5 Fr catheter. A 2.7 to 2.8 Fr microcatheter (Progreat, Terumo, Japan) was used to selectively cannulate tumor feeders. In cases where there were multifocal lesions in one lobe, the micro-catheter was positioned proximally in the feeding artery of a sector or a lobe. The emulsion consisted of 5-30 ml of lipiodol (Guerbet, Aulnay-sous-Bois, France) mixed with 40-60 ml Epirubicin (Hisun Pharmaceutical, Zhejiang, China), which was slowly injected through microcatheter under fluoroscopic-monitoring. The diameter of the index lesion in $\mathrm{cm}$ was multiplied by two to calculate the volume of iodized oil in $\mathrm{ml}$ used to make the emulsion. The entire emulsion was injected unless stasis was achieved or the portal vein around the tumor was visualized. This was followed with injection of particles such as 150-350 and 350-550 $\mu \mathrm{m}$ gelfoam particles (Alicon Pham SCI \&TEC, Hangzhou, China) or 100-300 and 300-500 $\mu \mathrm{m}$ embosphere particles (Biosphere Medical, Rockland, Massachusetts, USA) until stasis was achieved. In cases where the territory of more 
Table 1 Baseline characteristics of patients with HCC

\begin{tabular}{|c|c|}
\hline Characteristic & No. of Patient/Mean \\
\hline No. of patients & 29 \\
\hline Age (y) & $59.2 \pm 11.6$ \\
\hline Sex (male/female) & $26 / 3$ \\
\hline \multicolumn{2}{|l|}{ Etiology } \\
\hline Hepatitis B & 27 (93.1\%) \\
\hline Hepatitis C & $1(3.4 \%)$ \\
\hline ECOG performance status $(0 / 1 / 2 / 3 / 4)$ & $21 / 7 / 1 / 0 / 0$ \\
\hline Status 0 & $21(72.4 \%)$ \\
\hline Status 1 & $7(24.1 \%)$ \\
\hline Status 2 & $1(3.5 \%)$ \\
\hline Child-Pugh class (A/B/C) & $26 / 3 / 0$ \\
\hline A & 26 \\
\hline B & 3 \\
\hline \multicolumn{2}{|l|}{$\operatorname{AFP}(\mathrm{ng} / \mathrm{ml})$} \\
\hline$<10$ & $5(17.2 \%)$ \\
\hline $10-400$ & $15(51.7 \%)$ \\
\hline$>400$ & $9(31.0 \%)$ \\
\hline \multicolumn{2}{|l|}{ Prior hepatic resection } \\
\hline Yes & $7(24.1 \%)$ \\
\hline No & $22(75.9 \%)$ \\
\hline Index tumor numbers (per patient) & $3.22 \pm 2.20(1-5)$ \\
\hline Size mean + sd (range, in mm) & $28.1 \pm 24.4(10.0-136.3)$ \\
\hline \multicolumn{2}{|l|}{ Tumor pattern } \\
\hline Unifocal & 12 \\
\hline Multifocal & 39 \\
\hline \multicolumn{2}{|l|}{ Tumor location } \\
\hline Caudal lobe & 1 \\
\hline Left lobe & 14 \\
\hline Right lobe & 35 \\
\hline Border & 1 \\
\hline
\end{tabular}

than one vessel was treated, the emulsion was split between the receiving vessels at the discretion of angiographer. At the end point of cTACE based on fluoroscopy, CBCT was performed to assess the retention pattern of lipiodol.

\section{Cone beam computed tomography}

During a $10 \mathrm{~s}$ acquisition, 293 projection images were obtained with $180^{\circ}$ rotation $\left(20^{\circ} / \mathrm{s}\right)$. Cross-sectional images with $1.8 \mathrm{~mm}$ slice thickness and $512 \times 512 \times 512$ matrix size were reconstructed from the projections. Images were reconstructed within approximately two minutes.

\section{Image analysis}

The patterns of lipiodol retention by the tumor (s) on CBCT and fluoroscopy images were evaluated at the workstation (Advantage Workstation 4.3, GE Healthcare).
The retention patterns were classified by two experienced radiologists (14, 16 years experiences in interventional radiology) and decisions were reached by consensus. The retention patterns were classified as "complete" (more than $90 \%$ dense retention of the tumor, no peripheral defects), "moderate" (50-90\% dense retention, with or without peripheral defects), and "poor" with less than $50 \%$ dense retention of the tumor, with peripheral defects or no retention at all.

\section{Tumor response evaluation}

All study patients were evaluated by baseline CE-CT or CE-MRI within 1 month before cTACE. The index tumors were identified and baseline measurements made. The follow-up CE-CT or CE-MRI scan was performed 4-6 weeks after cTACE. Tumor response was assessed on the follow-up images according to the mRECIST criteria [17]. Unidimensional measurement of the longest diameter was recorded for each index tumor, which was selected according to mRECIST standards. Tumor responses were evaluated by a third experienced radiologist (16 years experiences in abdominal imaging) who was blinded to the study data separately.

Response was defined as follows: "complete response" (CR) as disappearance of all intratumoral enhancement; "partial response" (PR) as $<30 \%$ decrease in diameters of enhancing tumor from baseline; "progressive disease" (PD) as $>20 \%$ increase in diameters of enhancing tumor from baseline; and "stable disease" (SD) as all other tumors.

\section{Statistical analysis}

The patient demographics and tumor data were recorded in a secure database and expressed as mean and median. To assess risk factors for tumor response, univariate and multivariate analyses were performed through binary logistic regression. All analyses were performed by an independent investigator using SPSS (version 18.0). P value of less than 0.05 was set as significant for all analyses.

\section{Results and discussion}

\section{Lipiodol retention pattern and tumor response}

The pattern of lipiodol retention was different between fluoroscopy and CBCT (Figs. 1 and 2). In fluoroscopic images of 51 tumors, the complete, moderate and poor classes of lipiodol retention patterns were 23 (45.1\%), 19 (37.3\%), and 9 (17.6\%), respectively. In CBCT images, however, the results were 39 (76.5 \%), 11 (21.6\%), and $1(2.0 \%)$, respectively. Of all the tumors, $78.4 \%$ $(n=40)$ showed a CR, and $15.7 \%(n=8), 2.0 \%(n=1)$, and $3.9 \%(n=2)$ showed PR, SD, and PD.

In the "excellent" retention tumors by CBCT, 34/39 tumors $(87.2 \%)$ showed $C R$ and 5/39(12.8 \%) tumors showed PR. By comparison, in the "excellent" retention 

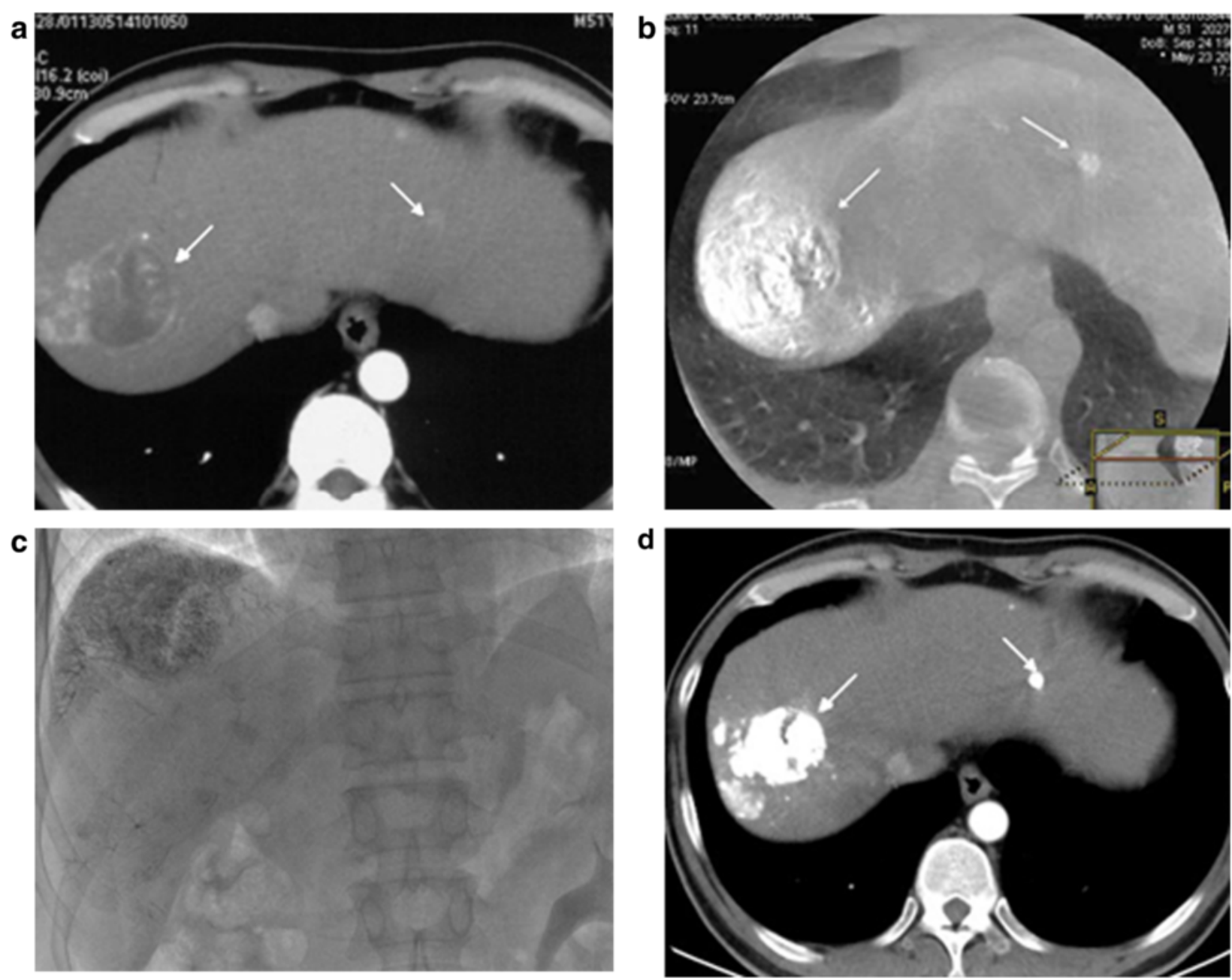

Fig. 1 A 52-year-old male with multiple HCCs. a Preprocedure arterial phase contrast-enhanced CT shows index tumors in segments II and VII (arrows); b CBCT immediately after TACE showed these two tumor with complete lipiodol retention pattern concordance with CE-CT (arrows); c On the fluoroscopy image, complete lipiodol retention pattern is well shown on the large tumor in segment VII, but no retention in the small tumor in segment II. d Postprocedure 1 month follow-up contrast-enhancement CT shows both tumors without contrast enhancement (arrows) representing complete response

tumors by fluoroscopy group, 18/23(78.3 \%) showed CR response, while $16 / 19$ (84.2 \%) "moderate" retention tumors and 6/9 (66.7\%) "poor" retention tumors still showed CR response. The pattern of lipidol retention by $\mathrm{CBCT}$ versus fluoroscopy and the number of tumors which demonstrated completed vs partial response, stable disease, or progression of disease for $\mathrm{CBCT}$ versus fluoroscopy are listed in Table 2.

\section{Predictors of response}

The accuracy for predicting tumor response by assessing the accumulation of iodized oil shows significant difference between the CBCT $\left(A_{z}=0.75\right)$ and fluoroscopy $\left(A_{z}=0.54\right)$. The correlation between tumor response was stronger with CBCT classification than fluoroscopy classification. Thirty-four among 39 tumors
$(87.2 \%)$ in the "complete" retention tumors by CBCT showed CR. By comparison, in the classification based on the fluoroscopy, 18 tumors (78.3 \%) out of 23 "complete" lipiodol retention class showed CR response.

The correlation of patient, tumor, and lipiodol retention pattern variables and CR by univariate and multivariate analyses are reported in Tables 3 and 4. Multivariate analysis suggests that lipiodol retention pattern by $\mathrm{CBCT}$ and tumor size are independent predictors of achieving a complete radiologic response (OR 19.17, $p=0.01$ and OR $0.88, p=0.04$ respectively).

\section{Discussion}

Earlier determination of the tumor response after cTACE is essential in decision making for application of additional treatments in patients with HCC. 
a

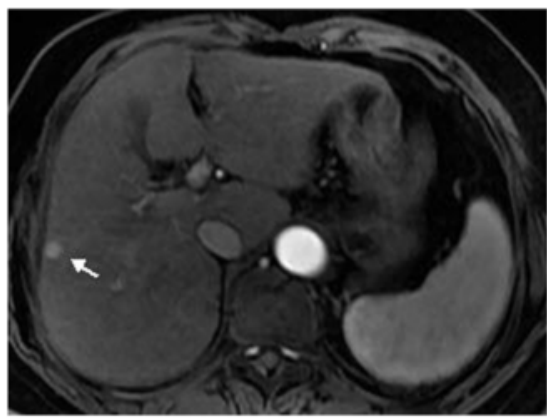

c

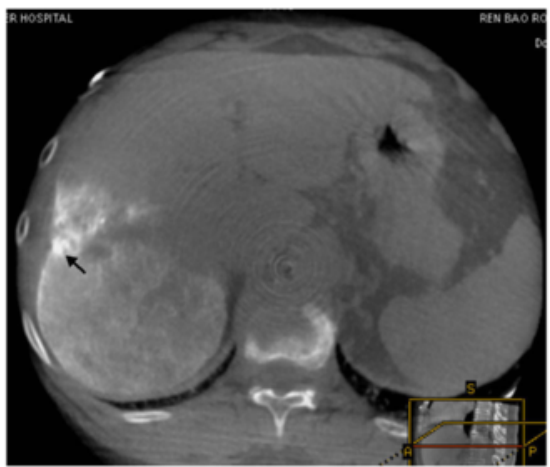

e

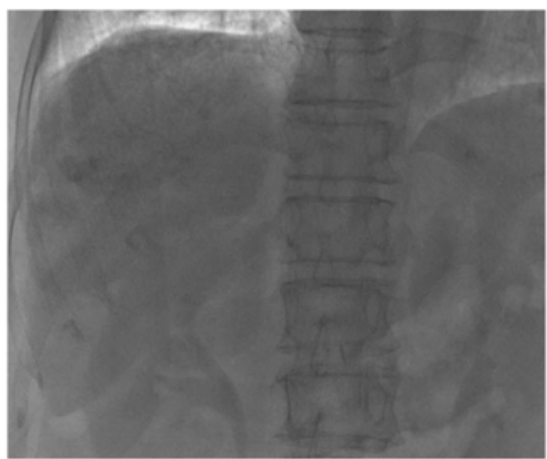

g

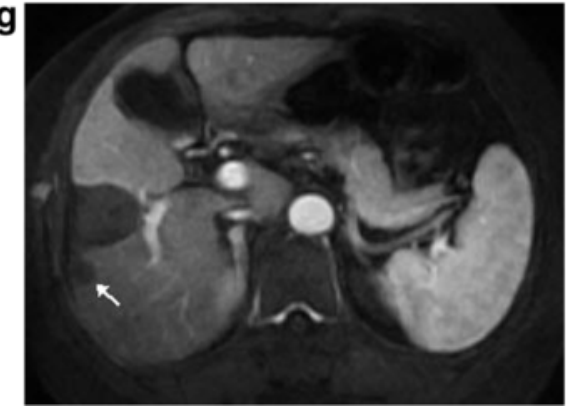

b

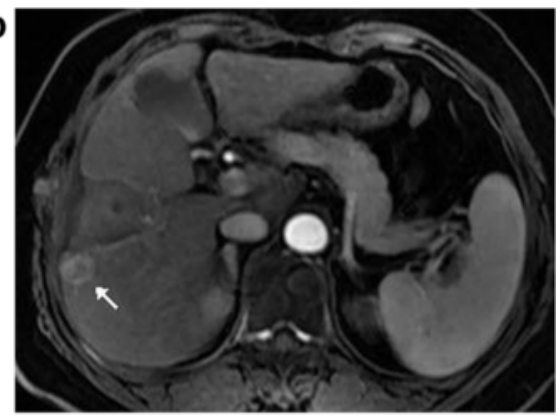

d

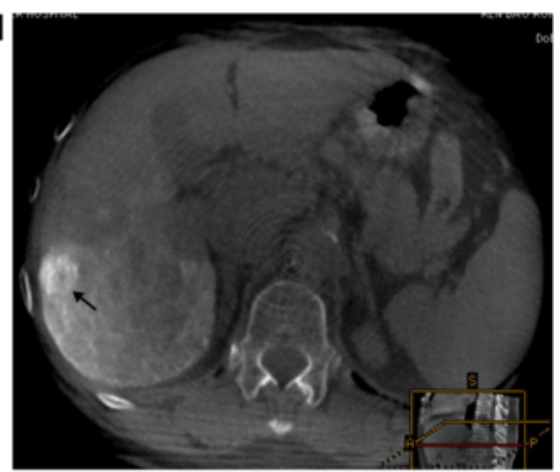

f

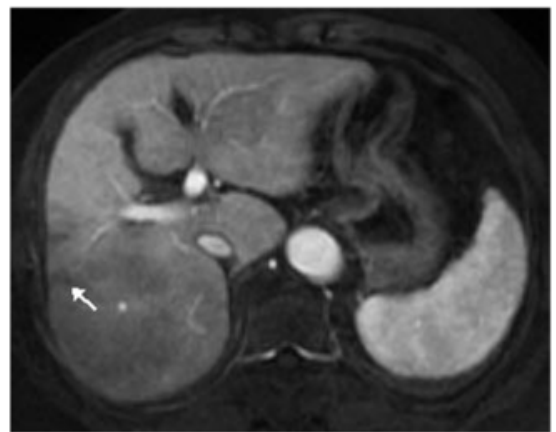

Fig. 2 A 56-year-old female with multiple HCCs and prior history of radiofrequency ablation. a, b Preprocedure arterial phase contrast-enhanced MRI shows two tumors adjacent to the ablation margin in the right lobe (arrows); c, $\mathbf{d}$ Cone beam CT immediately after completion of TACE showed these two tumors with complete lipiodol retention pattern without defect concordant with MRl; e On the fluoroscopy image, tumor lipiodol retention was not clearly seen. $\mathbf{f}, \mathbf{g}$ Postprocedure 1 month follow-up contrast-enhancement MRI showed necrosis without contrast enhancement in both tumors (arrows) representing complete response

Histopathologic examination for the determination of tumor response after transarterial chemoembolization is neither feasible nor acceptable. Imaging techniques, such as 4-6 weeks follow-up contrast-enhanced CT and MR imaging are widely used to evaluate the therapeutic effect $[17,18]$. For both EASL and mRECIST criteria, measurement of enhanced tumor portion rather than the total visible tumor size has been used to 
Table 2 Lipiodol retention variables and tumor response

\begin{tabular}{lllll}
\hline Variable & \multicolumn{3}{l}{ Response } \\
\hline Fluoroscopy & PD & SD & PR & CR \\
Excellent (23) & 0 & 0 & 5 & 18 \\
Moderate (19) & 1 & 0 & 2 & 16 \\
Poor (9) & 1 & 1 & 1 & 6 \\
Lip-CBCT & & & & \\
Excellent (39) & 0 & 0 & 5 & 34 \\
Moderate (11) & 1 & 1 & 3 & 6 \\
Poor (1) & 1 & 0 & 0 & 0 \\
\hline
\end{tabular}

evaluate tumor response in HCC patients after cTACE $[17,19]$. Based on this post-procedure assessment imaging, a repeat cTACE or tumor ablation would be needed in case there was residual viable tumors were identified.

Tumor progression for the incompletely treated tumors could be expected during this time interval. Given this risk, some investigators have reported the contrastenhanced ultrasonography (US) performed at least two and seven days after cTACE could be similar to the dynamic follow-up CT and be predictive of tumor outcome [20]. Some clinical studies have shown the ability of diffusion-weighted MR imaging to help quantify tumor necrosis after transcatheter liver-directed therapy [21, 22]. However, MR or US imaging are separate tests and require logistics for scheduling. Intraprocedural image monitoring is important to determine the endpoint of CTACE and application of additional treatment if

Table 3 Univariate analysis of predictors of CR

\begin{tabular}{llll}
\hline Factors & OR & $95 \% \mathrm{Cl}$ & $p$ \\
\hline Patients and tumor variables & & & \\
Sex (male/female) & 0.73 & $(0.51,1.04)$ & 0.01 \\
Age(y) $\geq 60$ vs. $<60$ & 2.07 & $(0.46,9.40)$ & 0.48 \\
Size $\geq 30 \mathrm{~mm}$ vs. $<30 \mathrm{~mm}$ & 0.32 & $(0.08,1.32)$ & 0.35 \\
Location & 1.01 & $(0.25,4.12)$ & 1.00 \\
Left vs. Right & & & \\
Unifocal vs. Multifocal & 0.32 & $(0.04,2.88)$ & 0.29 \\
Child-Pugh & 0.83 & $(0.17,4.01)$ & 0.82 \\
$\begin{array}{l}\text { A vs. B } \\
\text { AFP ( } \geq 400 \text { vs. < 400) }\end{array}$ & 4.07 & $(0.46,35.75)$ & 0.25 \\
$\begin{array}{l}\text { Prior hepatic resection } \\
\text { Yes vs. No }\end{array}$ & 0.57 & $(1.02,1.30)$ & 1.15 \\
Lipiodol retention on Fluoroscopy & 0.76 & $(0.22,2.64)$ & 0.75 \\
$\begin{array}{l}\text { Excellent vs. Moderate and Poor } \\
\text { Lipiodol retention on CBCT }\end{array}$ & 5.17 & $(1.13,23.55)$ & 0.03 \\
$\begin{array}{l}\text { Excellent vs. Moderate and Poor } \\
\text { Etiology }\end{array}$ & & & \\
Hepatitis B virus vs. negative & 2.19 & $(0.56,8.61)$ & 0.27 \\
\hline
\end{tabular}

Table 4 Multivariate models of predictors of CR

\begin{tabular}{lrll}
\hline Model & OR & $95 \% \mathrm{Cl}$ & $p$ \\
\hline Tumor Size & 0.88 & $(0.01,0.90)$ & 0.04 \\
Lipiodol retention in Lip-CBCT & 19.17 & $(1.87,196.49)$ & 0.01 \\
Excellent vs. Moderate and Poor & & & \\
\hline
\end{tabular}

needed while in the angiography suite. Utilization of CBCT performed at the time of CTACE for prediction of tumor response obviates the need for additional testing.

CBCT with flat-panel detector acquired during the CTACE procedure has been shown to have several advantages such as identifying the tumor feeding arteries, occult lesions, cyctic artery, etc. [23, 24]. Loffroy et al. showed dual-phase CBCT can be used to predict the short term response to TACE with drug-eluting beads [25].

cTACE with lipiodol is widely used and has comparable efficacy to DEB-TACE [26]. Lipiodol not only acts as a carrier of chemotherapeutic agents, but its use has yielded fairly beneficial therapeutic results. It exposes the tumor to high concentrations of the chemotherapeutic agents for a prolonged period of time, while minimizing systemic toxicity [27]. In this study we demonstrated the lipiodol retention pattern on CBCT immediately after CTACE can be used to predict the short-term tumor response at 4-6 weeks follow-up in HCC patient by mRECIST.

Lipiodol retention pattern after cTACE on follow-up $\mathrm{CT}$ imaging has been shown to be a prognostic marker $[8,18,28]$. Multidetector computed tomography (MDCT) can evaluate lipiodol retention after cTACE [14], however, the exact method for assessing lipiodol retention is to perform the post-procedural CT directly after cTACE and the patient must be transferred from the angiography suite to the CT scanner. Despite advances in the angiography unit, fluoroscopic imaging may not accurately show lipiodol retention pattern in the tumor because it cannot provide volumetric information such as CBCT [11]. A combined CT-angiography system with a stand alone CT scanner is useful to evaluate the lipiodol retention pattern; however, the system is expensive and requires a large room [9].

In this study, we have demonstrated that CBCT imaging performed during CTACE is superior to fluoroscopic imaging for assessing the lipiodol retention pattern in $\mathrm{HCC}$ tumors. A theoretical downside may be that $\mathrm{CBCT}$ may be less sensitive given its inferior spatial and contrast resolution compared with conventional CT [29]. However, it offers the advantage of imaging during a cTACE procedure without the necessity of transferring the patient. In one study, Rongxin Chen et al. have reported that CBCT imaging has a similar capability to assess Lipiodol retention as MDCT [14]. Correlation between contrast retention pattern in liver tumors detected on intraprocedural CT images during transcatheter bland embolization and 
tumor response has been reported [30]. Strong correlation between lipiodol retention on intraprocedural CBCT imaging and tumor response in HCC is shown with the help of three dimensional quantification software [15]. Our comparable results showing strong correlations could also be drawn with such software.

Assessing lipiodol retention pattern by $\mathrm{CBCT}$ during the CTACE procedure Provides near real-time feed-back [14] before the patient leaves the angiography suite and enables the operator to set a more accurate endpoint and perform additional treatment if necessary.

This study has limitations such as small size of the study group, its retrospective design and lack of a control group. Due to the small size of our group, interpretation of CT and MRI studies obtained after CTACE and $\mathrm{CBCT}$ images are prone to observer bias. The one-month follow-up treatment response evaluation may be inferior to the more commonly practiced 2-3 month follow-up treatment response evaluation. This might have introduced an element of bias. Our results need to be validated further with larger and better designed studies.

\section{Conclusion}

In conclusion, during cTACE for HCC tumors cone beam $\mathrm{CT}$ is more accurate in detection of lipiodol retention pattern compared to fluoroscopy. The pattern of lipiodol retention assessed by CBCT can serve as a prognostic indicator of short-term response and could be a reliable intraprocedural monitoring modility during CTACE.

\section{Abbreviations \\ CBCT: Cone beam computed tomography; CR: Complete response; $C T$ : Computer tomography; CTACE: Conventional transarterial chemoembolization; EASL: European Association for the Study of the Liver; ECOG: Eastern Cooperative Oncology Group; HCC: Hepatocellular carcinoma; INR: International normalized ratio; MDCT: Multidetector computer tomography; mRECIST: Modified response evaluation criteria in solid tumors; MRI: Magnetic resonance imaging; PD: Progressive disease; PR: Partial response; SD: Stable disease; US: Ultrasonography}

\section{Acknowledgements}

The abstract of this paper has been accepted for Oral Presentation at GEST 2016 US.

\section{Funding}

This project was supported by NSFC (81471759), Technology Foundation for Selected Overseas Chinese Scholar; Beijing High Level Medical Personal Fund (2013-3-084).

\section{Availability of data and materials}

Not applicable.

\section{Authors' contributions}

XW proposed the study. JH and XW performed research and wrote the first draft. MM revised the first draft and polished the language. XW collected and analyzed the data. All authors contributed to the design and interpretation of the study and to further drafts. XW is the guarantor. All authors read and approved the final manuscript.

\section{Authors' information}

Not applicable.

\section{Competing interests}

The authors declare that they have no competing interests.

\section{Consent for publication}

Not applicable.

Ethics approval and consent to participate

This study was approved by the Ethics Committee of Peking University Cancer Hospital \& Institute (No. 2014KT90).

\section{Author details}

${ }^{1}$ Department of Interventional Radiology, Peking University Cancer Hospital \& Institute, Key laboratory of Carcinogenesis and Translational Research (Ministry of Education), Beijing 100142, China. ' ${ }^{2}$ Department of Radiology, Interventional Radiology Service, Memorial Sloan-Kettering Cancer Center, 1275 York Ave, New York 10021, NY, USA. ${ }^{3}$ Department of Epidemiology and Biostatistics, Peking University Sixth Hospital, Beijing 100191, China.

Received: 29 July 2016 Accepted: 10 September 2016

Published online: 03 October 2016

\section{References}

1. Parkin DM, Bray F, Ferlay J, Pisani P. Global cancer statistics, 2002. CA Cancer J Clin. 2005;55(2):74-108.

2. Takayasu K. Transcatheter arterial chemoembolization for unresectable hepatocellular carcinoma: recent progression and perspective. Oncology. 2013;84 Suppl 1:28-33.

3. Murata S, Mine T, Sugihara F, Yasui D, Yamaguchi H, Ueda T, Onozawa S, Kumita SI. Interventional treatment for unresectable hepatocellular carcinoma. World Journal of Gastroenterology: WJG. 2014;20(37):13453-65.

4. Lo CM, Ngan H, Tso WK, Liu CL, Lam CM, Poon RT, Fan ST, Wong J. Randomized controlled trial of transarterial lipiodol chemoembolization for unresectable hepatocellular carcinoma. Hepatology (Baltimore, Md). 2002; 35(5):1164-71

5. Lencioni R, Petruzzi P, Crocetti L. Chemoembolization of hepatocellular carcinoma. Semin Interv Radiol. 2013;30(1):3-11.

6. Idee JM, Guiu B. Use of Lipiodol as a drug-delivery system for transcatheter arterial chemoembolization of hepatocellular carcinoma: a review. Crit Rev Oncol Hematol. 2013;88(3):530-49.

7. Yoshimitsu K. Transarterial chemoembolization using iodized oil for unresectable hepatocellular carcinoma: perspective from multistep hepatocarcinogenesis. Hepatic Medicine: Evidence and Research. 2014;6:89-94.

8. Monsky WL, Kim I, Loh S, Li CS, Greasby TA, Deutsch LS, Badawi RD. Semiautomated segmentation for volumetric analysis of intratumoral ethiodol uptake and subsequent tumor necrosis after chemoembolization. AJR Am J Roentgenol. 2010;195(5):1220-30.

9. Takayasu K, Muramatsu Y, Maeda T, Iwata R, Furukawa H, Muramatsu Y, Moriyama N, Okusaka T, Okada S, Ueno H. Targeted Transarterial Oily Chemoembolization for Small Foci of Hepatocellular Carcinoma Using a Unified Helical CT and Angiography System: Analysis of Factors Affecting Local Recurrence and Survival Rates. AJR Am J Roentgenol. 2001;176:681-8.

10. Fujita T, Ito K, Tanabe M, Yamatogi S, Sasai H, Matsunaga N. lodized oil accumulation in hypervascular hepatocellular carcinoma after transcatheter arterial chemoembolization: comparison of imaging findings with CT during hepatic arteriography. Journal of Vascular and Interventional Radiology: JVIR. 2008;19(3):333-41.

11. Jeon UB. lodized oil uptake assessment with cone-beam CT in chemoembolization of small hepatocellular carcinomas. World J Gastroenterol. 2009;15(46):5833.

12. Choi Bl, Kim HC, Han JK, Park JH, Kim YI, Kim ST, Lee HS, Kim CY, Han MC. Therapeutic Effect of Transcatheter Oily Chemoembolization Therapy for Encapsulated Nodular Hepatocellular Carcinoma: CT and Pathologic Findings. Radiology. 1992;182(3):709-13.

13. Wallace MJ, Kuo MD, Glaiberman C, Binkert CA, Orth RC, Soulez G, Technology Assessment Committee of the Society of Interventional R. Threedimensional C-arm cone-beam CT: applications in the interventional suite. Journal of Vascular and Interventional Radiology: JVIR. 2008;19(6):799-813.

14. Chen R, Geschwind JF, Wang Z, Tacher V, Lin M. Quantitative assessment of lipiodol deposition after chemoembolization: comparison between conebeam $\mathrm{CT}$ and multidetector $\mathrm{CT}$. Journal of Vascular and Interventional Radiology: JVIR. 2013;24(12):1837-44. 
15. Wang Z, Chen R, Duran R, Zhao Y, Yenokyan G, Chapiro J, Schernthaner R, Radaelli A, Lin M, Geschwind JF. Intraprocedural 3D Quantification of Lipiodol Deposition on Cone-Beam CT Predicts Tumor Response After Transarterial Chemoembolization in Patients with Hepatocellular Carcinoma. Cardiovasc Intervent Radiol. 2015;38(6):1548-56.

16. Ikeda M, Arai Y, Park SJ, Takeuchi Y, Anai H, Kim JK, Inaba Y, Aramaki T, Kwon SH, Yamamoto S, et al. Prospective study of transcatheter arterial chemoembolization for unresectable hepatocellular carcinoma: an Asian cooperative study between Japan and Korea. Journal of Vascular and Interventional Radiology: JVIR. 2013;24(4):490-500.

17. Lencioni R, Llovet JM. Modified RECIST (mRECIST) assessment for hepatocellular carcinoma. Semin Liver Dis. 2010;30(1):52-60.

18. Herber S, Biesterfeld S, Franz U, Schneider J, Thies J, Schuchmann M, Duber C, Pitton MB, Otto G. Correlation of multislice $C T$ and histomorphology in HCC following TACE: predictors of outcome. Cardiovasc Intervent Radiol. 2008;31(4): 768-77.

19. Bruix J, Sherman M, Llovet JM, Beaugrand M, Lencioni R, Burroughs AK, Christensen E, Pagliaro L, Colombo M, Rodes J. Clinical management of hepatocellular carcinoma. Conclusions of the Barcelona-2000 EASL conference. European Association for the Study of the Liver. J Hepatol. 2001;35(3):421-30.

20. Kono Y, Lucidarme O, Choi SH, Rose SC, Hassanein TI, Alpert E, Mattrey RF. Contrast-enhanced ultrasound as a predictor of treatment efficacy within 2 weeks after transarterial chemoembolization of hepatocellular carcinoma. Journal of Vascular and Interventional Radiology: JVIR. 2007;18(1 Pt 1):57-65.

21. LE Kamel IR, Reyes DK, Zahurak M, Bluemke DA, Geschwind JF. Unresectable Hepatocellular Carcinoma: Serial Early Vascular and Cellular Changes after Transarterial Chemoembolization as Detected with MR Imaging. Radiology. 2009;250(2):466-73.

22. Chiao-Yun Chen M, Chun-Wei Li P, Yu-Ting Kuo M, Twei-Shiun Jaw M, Ding-Kwo Wu M, Jo-Chi Jao P, Jui-Sheng Hsu M, Gin-Chung Liu M. Early Response of Hepatocellular Carcinoma to Transcatheter Arterial Chemoembolization Choline Levels and MR Diffusion Constants-Initial Experience. Radiology. 2006:239(2):448-56.

23. Deschamps F, Solomon SB, Thornton RH, Rao P, Hakime A, Kuoch V, de Baere T. Computed Analysis of Three-Dimensional Cone-Beam Computed Tomography Angiography for Determination of Tumor-Feeding Vessels During Chemoembolization of Liver Tumor: A Pilot Study. Cardiovasc Intervent Radiol. 2010;33(6):1235-42

24. Miyayama S, Yamashiro M, Okuda M, Yoshie Y, Sugimori N, Igarashi S, Nakashima Y, Matsui O. Usefulness of cone-beam computed tomography during ultraselective transcatheter arterial chemoembolization for small hepatocellular carcinomas that cannot be demonstrated on angiography. Cardiovasc Intervent Radiol. 2009;32(2):255-64.

25. Loffroy R, Favelier S, Cherblanc V, Estivalet L. C-arm dual-phase cone-beam CT: a revolutionary real-time imaging modality to assess drug-eluting beads TACE success in liver cancer patients. Quant Imaging Med Surg. 2013;3(4):196-9.

26. Johannes Lammer KM, Malagari K, Vogl T, Pilleul F, Denys A, Watkinson A, Pitton M, Sergent G, Pfammatter T, Terraz S, Benhamou Y, Avajon Y, Gruenberger T, Pomoni M, Langenberger H, Schuchmann M, Dumortier J, Mueller C, Chevallier P, Lencioni R, On Behalf of the PRECISION V Investigators. Prospective Randomized Study of Doxorubicin-Eluting-Bead Embolization in the Treatment of Hepatocellular Carcinoma: Results of the PRECISION V Study. Cardiovasc Intervent Radiol. 2009;33:41-52.

27. Nakao N, Uchida H, Kamino K, Nishimura Y, Ohishi H, Takayasu Y, Nakamura H, Kuroda C, Fujita M, Yoshioka H, et al. Effectiveness of Lipiodol in transcatheter arterial embolization of hepatocellular carcinoma. Cancer Chemother Pharmacol. 1992:31(Suppl):S72-6

28. Mondazzi L, Bottelli R, Brambilla G, Rampoldi A, Rezakovic I, Zavaglia C, Alberti A, Ideo G. Transarterial oily chemoembolization for the treatment of hepatocellular carcinoma: a multivariate analysis of prognostic factors. Hepatology (Baltimore, Md). 1994;19(5):1115-23.

29. Tognolini A, Louie JD, Hwang GL, Hofmann LV, Sze DY, Kothary N. Utility of $\mathrm{C}$-arm CT in patients with hepatocellular carcinoma undergoing transhepatic arterial chemoembolization. Journal of Vascular and Interventional Radiology: JVIR. 2010;21(3):339-47.

30. Wang X, Erinjeri JP, Jia X, Gonen M, Brown KT, Sofocleous CT, Getrajdman Gl, Brody LA, Thornton RH, Maybody M, et al. Pattern of retained contrast on immediate postprocedure computed tomography (CT) after particle embolization of liver tumors predicts subsequent treatment response. Cardiovasc Intervent Radiol. 2013:36(4):1030-8.

\section{Submit your next manuscript to BioMed Central and we will help you at every step:}

- We accept pre-submission inquiries

- Our selector tool helps you to find the most relevant journal

- We provide round the clock customer support

- Convenient online submission

- Thorough peer review

- Inclusion in PubMed and all major indexing services

- Maximum visibility for your research

Submit your manuscript at www.biomedcentral.com/submit
Biomed Central 